\title{
A useful additional medial subbrow approach for the treatment of medial orbital wall fracture with subciliary technique
}

\author{
Seung Min Kim ${ }^{1}$, \\ Cheol Keun Kim ${ }^{1}$, \\ Dong In Jo', \\ Myung Chul Lee², \\ Ji Nam Kim ${ }^{2}$, \\ Hyun Gon Choi ${ }^{2}$, \\ Dong Hyeok Shin ${ }^{2}$, \\ Soon Heum Kim ${ }^{1}$
}

${ }^{1}$ Department of Plastic and Reconstructive Surgery, Konkuk University Chungju Hospital, Konkuk University School of Medicine, Chungju; ${ }^{2}$ Department of Plastic and

Reconstructive Surgery, Konkuk University Seoul Hospital, Konkuk University School of Medicine, Seoul, Korea

\begin{abstract}
Background: To date, a variety of surgical approaches have been used to reconstruct the medial orbital wall fracture. Still however, there is still a controversy as to their applicability because of postoperative scars, injury of anatomical structures and limited visual fields. The purpose of this study was to introduce a useful additional medial subbrow approach for better reduction and securement more accurate implant pocket of medial orbital wall fracture with the subciliary technique.

Methods: We had performed our technique for a total of 14 patients with medial orbital wall fracture at our medical institution between January 2016 and July 2017. All fractures were operated through subciliary technique combined with the additional medial subbrow approach. They underwent subciliary approach accompanied by medial wall dissection using a Louisville elevator through the slit incision of the medial subbrow procedure. This facilitated visualization of the medial wall fracture site and helped to ensure a more accurate pocket for implant insertion.

Results: Postoperative outcomes showed sufficient coverage without displacement. Twelve cases of preoperative diplopia improved to two cases of postoperative diplopia. More than $2 \mathrm{~mm}$ enophthalmos was 14 cases preoperatively, improving to 0 case postoperatively. Without damage such as major vessels or extraocular muscles, enophthalmos was corrected and there was no restriction of eyeball motion.

Conclusion: Our ancillary procedure was useful in dissecting the medial wall, and it was a safe method as to cause no significant complications in our clinical series. Also, there is an only nonvisible postoperative scar. Therefore, it is a recommendable surgical modality for medial orbital wall fracture.
\end{abstract}

Keywords: Dissection / Fracture fixation / Orbit / Orbital fractures

\section{INTRODUCTION}

Orbital fractures commonly occur in the midface region, thus accounting for $13.3 \%$ of all facial bone fractures, with reported

\footnotetext{
Correspondence: Soon Heum Kim

Department of Plastic and Reconstructive Surgery, Konkuk University Chungju Hospital, Konkuk University School of Medicine, 82 Gugwon-daero, Chungju 27376, Korea

E-mail: plastic7@kku.ac.kr

Received April 10, 2019 / Revised April 20, 2019 / Accepted April 20, 2019
}

rates of isolated medial orbital wall fractures ranging from $0 \%$ to $55 \%$ [1]. Orbital medial wall fracture is difficult to correct and dissect because of its adjacent location to the globe, nerves and vessels, and the limited visual fields.

To date, a variety of surgical approaches have been used to reconstruct the medial orbital wall fracture. These include medial canthal incision (Lynch incision), transconjunctival incision, subciliary incision, intranasal approach, lid crease incision, and bicoronal incision. Of these, the transconjunctival approach 
leaves no visible scar and has a brief course but provides narrow operative exposure, possible postoperative conjunctival irritation and the potential for conjunctival granuloma [2]. Subciliary incision is a familiar method of accessing the lower half or lower two-thirds of the medial wall, but it is not easy to obtain full exposure of the operative field [3]. There is still a controversy as to their applicability because of postoperative scars, injury of anatomical structures and limited visual fields. Sufficient dissection of the fracture site is difficult with any incision method [4].

To date, a replacement has been performed rather than restoration to reconstruct orbital medial wall fracture, for which subperiosteal dissection is mandatory. When placing a large implant, even a small misstep in alignment will result in significant deviation of the plate from an anatomic reduction of the orbital size. Sufficient exposure and visualization of the medial wall are necessary to avoid this problem [5].

Given the above background, we have devised a useful additional procedure for dissecting the medial orbital wall fracture considering the anatomical characteristics of the orbit as there might be no clear method for periosteal dissection. The purpose of this study was to introduce a useful additional medial subbrow approach for better reduction and securement more accurate implant pocket of medial orbital wall fracture with the subciliary technique.

\section{METHODS}

\section{Patient}

We had performed our technique for a total of 14 patients with medial orbital wall fracture at our medical institution between January 2016 and July 2017.

Our clinical series of the patients is composed of seven with pure blow-out fracture and another seven with impure one. We therefore enrolled a total of 14 patients $(n=14)$ in the current study; it was approved by the Institutional Review Board of Konkuk University Hospital.

The patients were followed up during a median period of 9 months (range, 6-14 months). All the patients were preoperatively and postoperatively evaluated for enophthalmos, diplopia, the range of the motion of extraocular muscle (EOM) and the presence of concurrent ocular diseases on physical examination. Enophthalmos was measured with a Hertel exophthalmometer. The indications for the operation were as follows: persistent diplopia, decreased visual acuity, limited ocular muscle movement (EOM), enophthalmos of $\geq 2 \mathrm{~mm}$, bone defects of $\geq 2 \mathrm{~cm}^{2}$ on computed tomography (CT) scans.

\section{Surgical technique}

Surgery was performed by one surgeon (SHK). All the patients were laid in a supine position. Under general anesthesia, their facial region was disinfected and then covered with a sterilization wrap. To prevent the occurrence of corneal damages, we intraoperatively applied an eye protector to the patients. Surgery was performed using a subciliary technique.

Surgery was performed to reconstruct the fracture of the medial orbital wall using a subciliary incision. At the arcus marginalis, a periostotomy was performed. This was followed by the periosteal dissection on the orbital floor. A partial dissection of the medial orbital wall fracture was also performed. We performed partial dissection of the medial orbital wall up to anteriorly $3 / 4$ and inferiorly $1 / 4$ to $1 / 5$.

Our useful additional procedure was illustrated in Fig. 1. The orbital margin in the medial portion of subbrow was palpated to determine where the margin becomes sharp to blunt. Following injection of $2 \%$ lidocaine mixed with epinephrine for bleeding control, a 2-mm slit incision was performed on the medial side of the subbrow using a No. 11 blade. This point was transitional zone between the roof and medial wall of the orbit; it is characterized by a lack of abrupt concavity of the arcus marginalis and the connection to the medial orbital wall in a round-to-flat shape (Fig. 2).

The tip of the Metzenbaum was pushed into a slit incision to ensure the bony contact of the orbital margin. After the orbital margin was confirmed once again, an incision was made on the periosteum by Louisville elevator. Then, a subperiosteal dissection was performed using a sharp, flat edge of Louisville elevator perpendicular to Frankfurt line without resistance. This was followed by the connection of the subperiosteal plane of the previously dissected orbital floor (Fig. 3). Confirming the tip of the Louisville elevator, a fan-shaped dissection in the posterosuperior direction was performed while spatula into the subciliary technique maintains the subperiosteal space (Fig. 4). A fanshaped dissection proceeded with visual confirmation of the tip without resistance. This was accompanied by a stepwise reduction of the herniated soft tissue. The fracture site was dissected carefully, recognizing that the anterior ethmoidal artery coming out from the anterior ethmoidal foramen which is an average of $24 \mathrm{~mm}$ behind the anterior lacrimal crest [6].

Finally, a sufficient dissection of the medial orbital wall fracture was performed using our method.

After a sufficient dissection of the fracture site, Medpor Titan (Stryker, Kalamazoo, MI, USA) was fabricated depending on fracture size. And then, spatula supported the globe to maintain subperiosteal space. Subsequently, the soft tissue containing the globe was retracted using the Louisville elevator to the lateral 

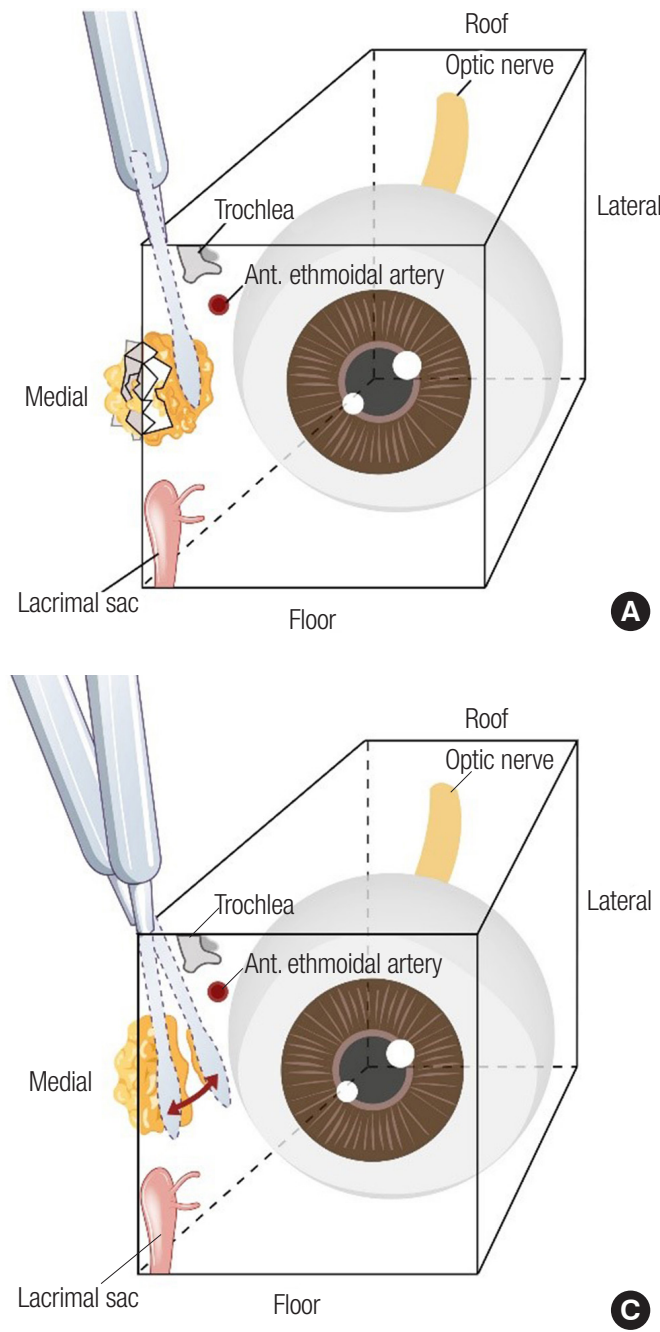
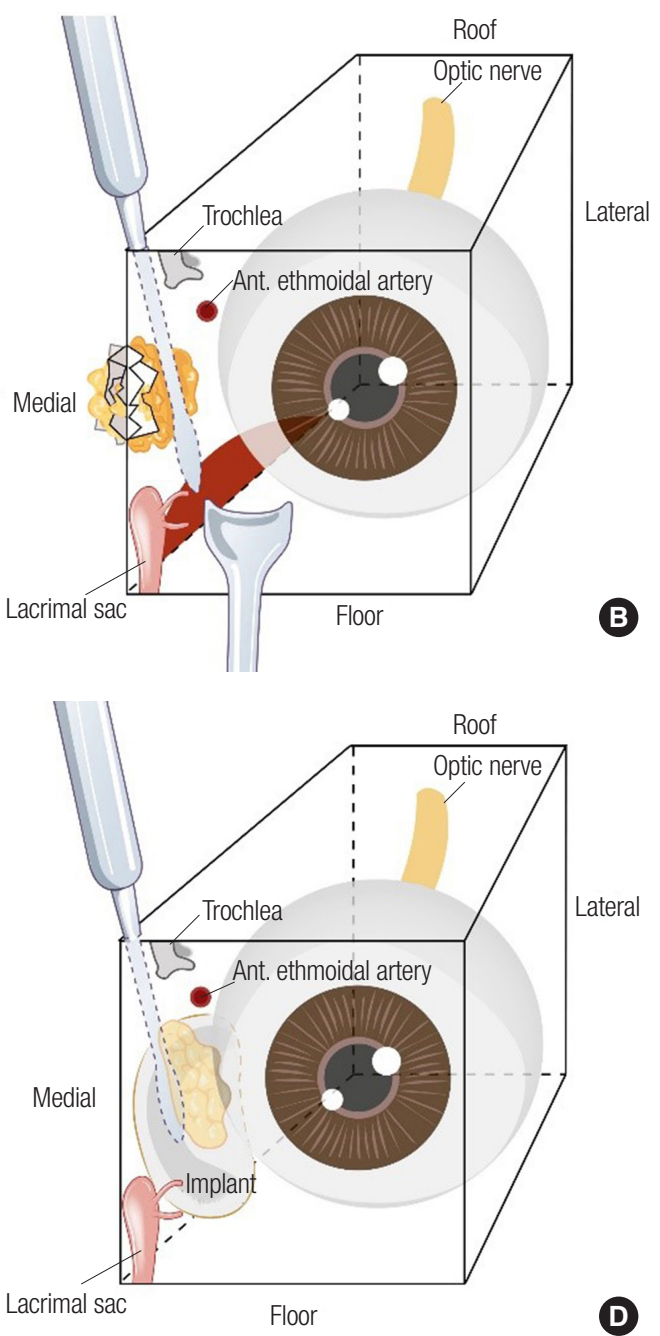

Fig. 1. Schematic illustration of surgical procedures. (A) Access to fracture site through the subbrow approach. (B) Connection of subperiosteal plane of the medial wall to the floor. Painted red zone indicates a partially dissected medial wall by subciliary technique. (C) Stepwise reduction of orbital herniated soft tissue. (D) Inserted implant on the medial side of the tip of the Louisville elevator. Ant., anterior.

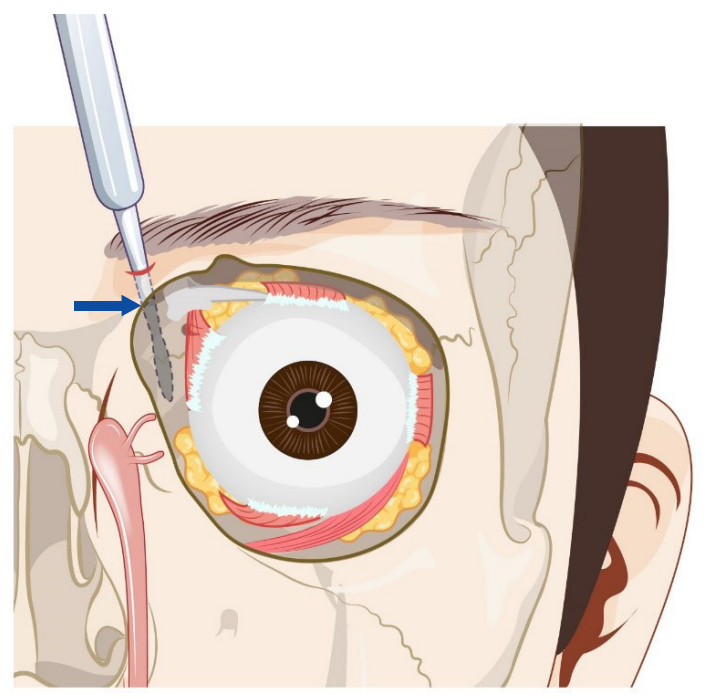

Fig. 2. The location of the transitional zone on the arcus marginalis (blue arrow).

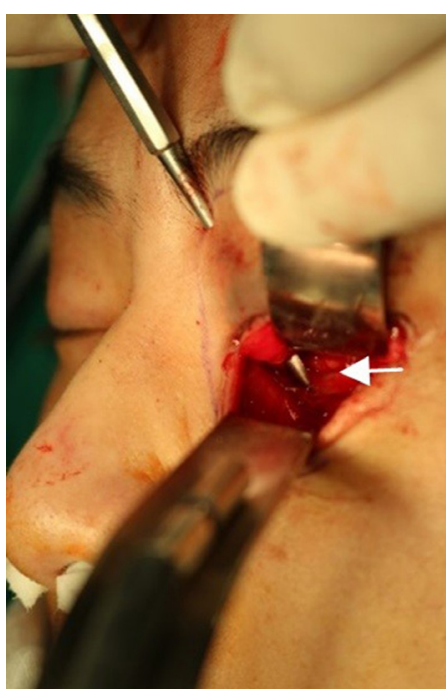

Fig. 3. Connection of subperiosteal plane to the floor. White arrow indicates the connection of the subperiosteal plane of medial wall to the floor. 
side. With this way, the implant pocket of the medial orbital wall is connected to the floor of the subperiosteal space and becomes the pathway of implant insertion. Then, Medpor Titan

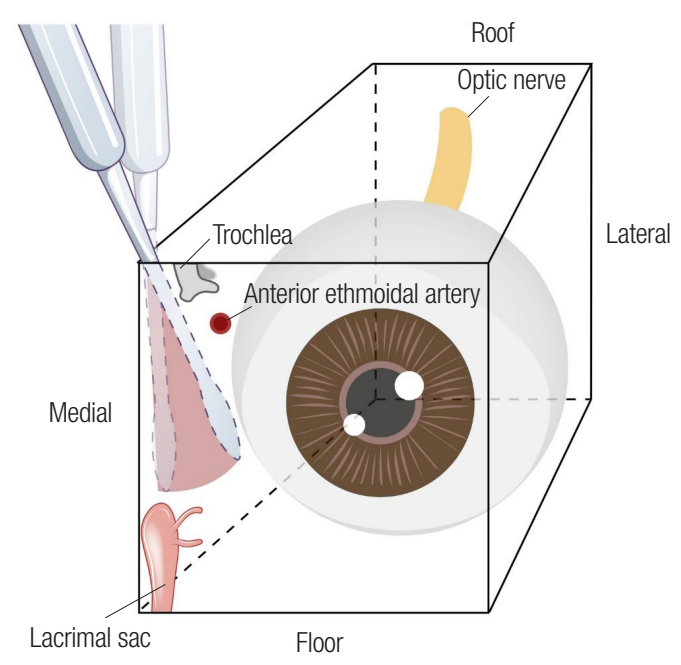

Fig. 4. A fan-shaped dissection of the medial wall. Painted red zone indicates a dissected range of medial wall by subbrow procedure. was inserted on the medial side of the tip of the Louisville elevator. Concurrently, the subperiosteal space was raised by the Louisville elevator and Medpor Titan was also inserted accordingly.

A forced duction test was performed to evaluate the EOM limitation and to prevent impingement of the soft tissue. The periosteum was closed using 4-0 Vicryl. After the insertion of a Penrose drain, the skin was sutured using 7-0 black silk. The puncture of subbrow was also closed using a 1-2 stitch of 7-0 black silk.

\section{RESULTS}

\section{Baseline characteristics of the patients}

Our clinical series of patients include 11 men and 3 women. The traffic accident was the most common cause of the injury. In addition, the mean time point of the surgery was 7.5 day of the onset of the injury. Of the preoperative symptoms, enophthalmos was seen in 14 patients (100\%) and diplopia was seen in 12 patients (83.3\%).
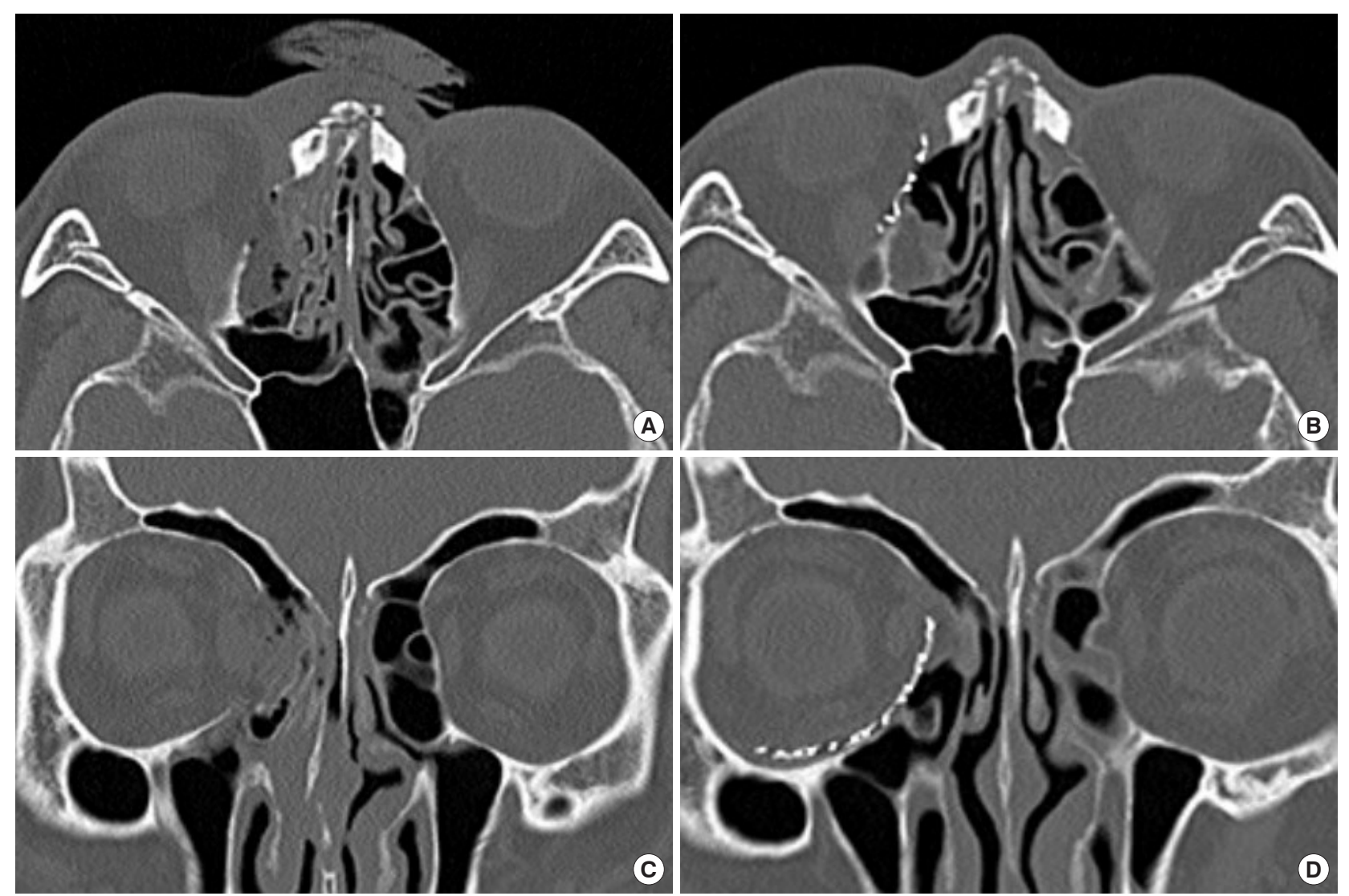

Fig. 5. Case 13. A 49-year-old man with right medial orbital wall fracture accompanied by nasal bone fracture. He underwent an operation using the useful additional procedure. (A, B) Preoperative and postoperative axial computed tomography (CT) scans demonstrating that the herniated orbital contents were restored and well placed Medpor Titan implant. (C, D) Preoperative and postoperative coronal CT scans demonstrating that the herniated orbital contents were restored and well placed Medpor Titan implant. 


\section{Postoperative outcomes}

All patients underwent a useful additional procedure in addition to the subciliary technique. They refused to take a medial canthal approach. In addition, we concomitantly performed our procedure with a subciliary technique, thus achieving a dissection of the fracture site more easily as compared with a single use of a subciliary technique or others. Postoperative outcomes showed sufficient coverage without displacement (Figs. 5, 6).

Twelve cases of preoperative diplopia improved to two cases of postoperative diplopia. Enophthalmos of $\geq 2 \mathrm{~mm}$ was 14 cases preoperatively, improving to 0 case postoperatively (Table 1). Lateral gaze limitation was two cases preoperatively, improving to full extraocular movement postoperatively. In our series, there were one case of hematoma leading to a revision surgery on postoperative day 3 and one case of ectropion. There were no complications such as infection, dislocation of the implant and visible scar.

\section{DISCUSSION}

Due to the difficulty of dissection, postoperative scar, limited visual fields, complications such as maxillary sinusitis, physiologic problems, packing, long learning curve and inconvenience of manipulation, the medial orbital wall fracture poses a surgical dilemma for surgeons $[7,8]$.

The difficulty of dissection is closely associated with the adjacent presence of the globe, nerves, vessels, lacrimal apparatus and medial canthal tendon. The supratrochlear nerve and vessels are present in the superior part of the orbital medial wall. In addition, the anterior and posterior ethmoidal arteries are distributed in the lamina papyracea of the medial orbital wall. Therefore, a periosteal dissection should be cautioned [9].

To date, subciliary incision has been used to reconstruct the medial orbital wall fracture. But this approach causes postoperative complications such as the formation of scars around the eyelid and ectropion. It is well known that complete dissection of the fracture site could be rarely achieved irrespective of types of incision in patients with medial orbital wall fracture. Patients with bursting fracture of the inferior or medial orbital wall are vulnerable to worsening of the severity of bony displacement. It is therefore difficult to reposition bone fragments to the original location in these patients $[10,11]$.
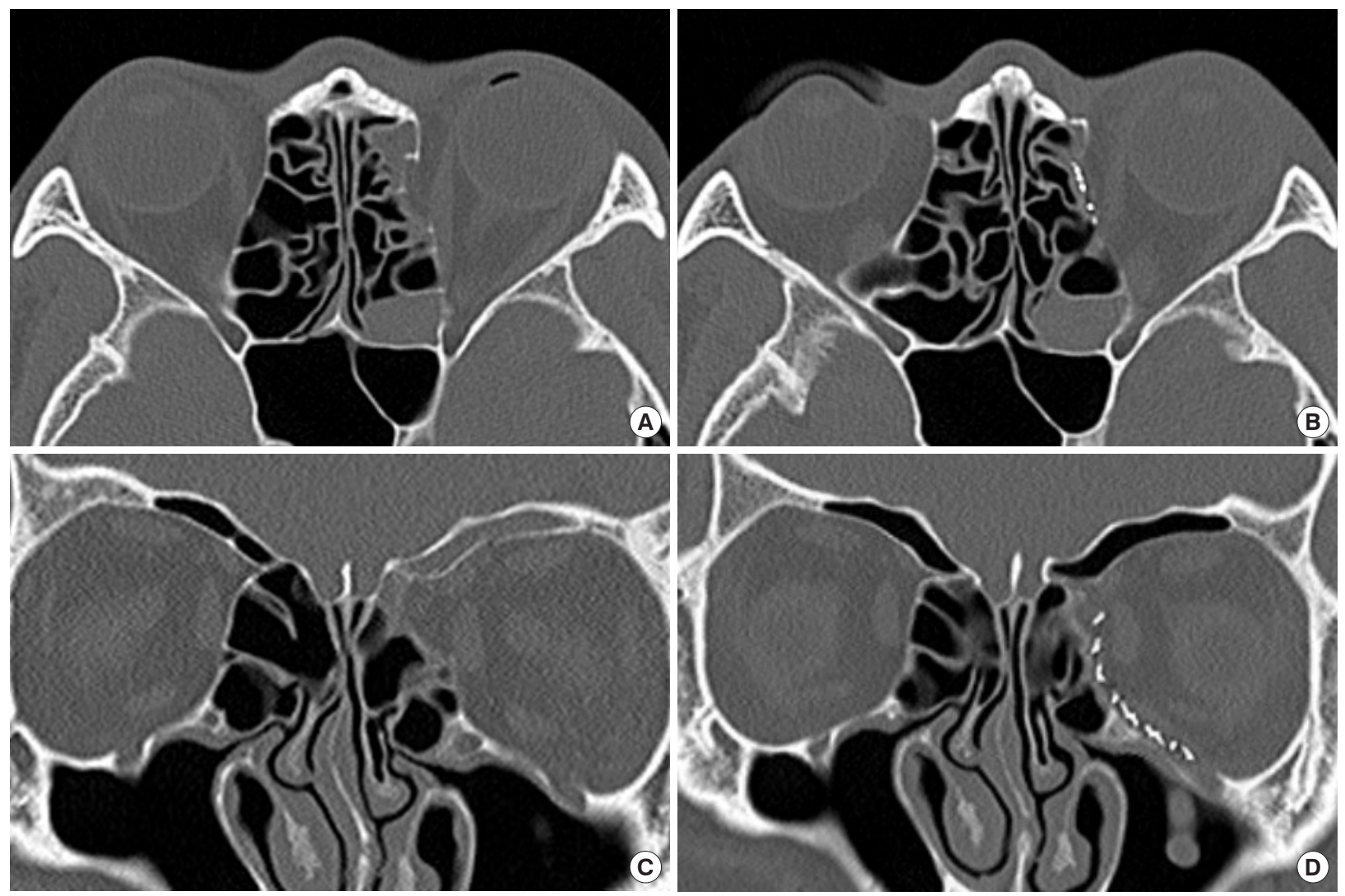

Fig. 6. Case 14. A 37-year-old man with left medial orbital wall fracture. He underwent an operation using the useful additional procedure. (A, B) Preoperative and postoperative axial computed tomography (CT) scans demonstrating that the herniated orbital contents were restored and well placed Medpor Titan implant. (C, D) Preoperative and postoperative coronal CT scans demonstrating that the herniated orbital contents were restored and well placed Medpor Titan implant. 
Table 1. Comparison of sequelae of patients

\begin{tabular}{|c|c|c|c|c|c|c|c|}
\hline \multirow{2}{*}{ Case } & \multirow{2}{*}{ Sex/age (yr) } & \multicolumn{2}{|c|}{ Diplopia } & \multicolumn{2}{|c|}{ Enophthalmos (mm) } & \multicolumn{2}{|c|}{ Extraocular movement } \\
\hline & & Preop & Postop & Preop & Postop & Preop & Postop \\
\hline 1 & $F / 67$ & Lateral (4 PD) & - & 2 & 0.5 & Full & Full \\
\hline 2 & $\mathrm{M} / 73$ & Lateral (8 PD) & Lateral (2 PD) & 2.5 & 1 & Full & Full \\
\hline 3 & $M / 51$ & $\begin{array}{l}\text { Lateral (4 PD) } \\
\text { Upward (2 PD) }\end{array}$ & - & 2 & 0 & Full & Full \\
\hline 4 & M/51 & - & - & 2.5 & 0.5 & Full & Full \\
\hline 5 & $\mathrm{~F} / 54$ & Lateral (4 PD) & - & 2 & 0.5 & Full & Full \\
\hline 6 & $\mathrm{M} / 19$ & Lateral (4 PD) & - & 2 & 0 & Lateral gaze limitation & Full \\
\hline 7 & $\mathrm{M} / 30$ & $\begin{array}{l}\text { Lateral (6 PD) } \\
\text { Upward (2 PD) }\end{array}$ & - & 2 & 0.5 & Full & Full \\
\hline 8 & $M / 25$ & Lateral (4 PD) & - & 2 & 0 & Lateral gaze limitation & Full \\
\hline 9 & $M / 20$ & - & - & 2.5 & 0 & Full & Full \\
\hline 10 & $\mathrm{M} / 48$ & Lateral (4 PD) & - & 2 & 0 & Full & Full \\
\hline 11 & $M / 54$ & Lateral (8 PD) & Lateral (2 PD) & 3 & 1 & Full & Full \\
\hline 12 & $\mathrm{~F} / 77$ & Lateral (6 PD) & - & 3 & 1 & Full & Full \\
\hline 13 & $\mathrm{M} / 49$ & Lateral (4 PD) & - & 2 & 0.5 & Full & Full \\
\hline 14 & $M / 37$ & $\begin{array}{l}\text { Lateral (4 PD) } \\
\text { Upward (2 PD) }\end{array}$ & - & 2 & 1 & Full & Full \\
\hline
\end{tabular}

Preop, preoperative; Postop, postoperative; F, female; M, male; PD, prism diopter.

A transcaruncular approach is a useful modality in that it does not cause visible scars. Due to relatively smaller visual fields, non-experienced surgeons encounter difficulty in placing the implant as well as performing dissection and reduction of fracture fragments. Furthermore, a longer learning curve is another disadvantage [12,13].

An endoscopic approach is used to reduce the orbital fracture via a nasal cavity and ethmoidal or maxillary sinus under the endoscopic guidance [14-17]. This ensures access to the medial and inferior orbital wall even without making an incision. An endoscopic approach is advantageous in not only preventing the separation between the fracture site and the periosteum but also minimizing the occurrence of bone defects. But its disadvantages include a restriction of visual fields and a difficulty in intraoperatively evaluating the degree of replacement. This lowers the accuracy of an anatomical reduction of the fracture. Moreover, it also requires a complete understanding of the anatomical structure and the skilled use of an endoscope. The applicability of an endoscopic approach is limited in patients with deviated nasal septum; these patients should receive additional surgeries such as septoplasty $[7,18]$. Its complications may include the entrapment of orbital contents between the orbital wall and a balloon or silastic sheet, orbital compression due to over-reduction, leakage of cerebrospinal fluid, the obstruction of sinus drainage pathways in the dependent sinus and infection [19]. Additionally, it also remains problematic that use of nasal packing materials, such as Nasopore (Polyganics, Groningen, the Netherlands) or Merocel (Medtronic Inc., Minneapolis, MN, USA) may also cause patient discomfort [16].

We have therefore devised a useful additional procedure considering the anatomical characteristics that the orbit and its lateral wall, roof, and floor have a sharply concave arcus marginalis. But the transitional zone between the orbital roof and medial wall showed a round-to-flat arcus marginalis [20]. Therefore, we have speculated that it would be easier to dissect the medial wall if we have access to the superomedial portion of the orbit.

For stability, a fan-shaped dissection was performed (Fig. 4), but with the spatula of the subciliary dissection, the subperiosteal space was maintained while the resistance was detected with direct visual confirmation. Dissection and orbital soft tissue reduction were performed in a stepwise manner while detecting resistance. Since the orbital medial wall is flat and the lateral wall is conical, there is little concern about injury to the medial rectus muscle.

For convenience, the learning curve is relatively shorter because slit incision was performed and the procedure is relatively simpler.

For efficacy, while floor dissection was also performed with a subciliary approach, at least some degree of orbital medial wall area was secured. In this regard, a fan-shaped dissection was performed in posterosuperior direction to expose sufficient fracture sites. This is essential for ensuring that there is suffi- 
cient and secure space to insert the implant. Because the implant is inserted in the medial part of the tip while looking directly at the tip of the Louisville elevator, the implant can be positioned properly.

Advantages of our additional procedure are as follows. First, herniated orbital soft tissue reduction is easier and definite. Second, almost no visible scars are left except for subciliary incision. An only puncture wound is needed during surgery. Third, the extent of dissection is relatively larger. With an ability to perform a fan-shaped dissection, a sufficient extent of dissection of the medial orbital wall could be achieved. Fourth, a more stable implant setting is achieved. A sufficient dissection of the fracture site makes it easier to place and fix an implant to the desired location more easily. Fifth, surgery can be performed simultaneously with an accurate replacement.

Based on the above advantages, we propose that our technique be used to achieve a sufficient dissection of the medial orbital wall. A single use of conventional approaches for the medial orbital wall fracture often achieves an insufficient dissection of the medial orbital wall. Therefore, we have shown that concomitant use of our procedure was useful in resolving the above problem. Our procedure is a valuable option in that it is reproducible and convenient, and causes almost no complications. The globe is surrounded by the condensation of fascia with a sufficient degree of strength and thickness, and it is composed of Tenon's capsule [21]. A sufficient dissection of the fracture site without hemorrhage was possible. Bone fragments can also be successfully dissected without breakage through a piece-by-piece dissection of them.

An accurate reconstruction of the medial orbital fracture poses a surgical dilemma for plastic surgeons because of disadvantages of conventional methods of dissection, limited visual fields and the presence of vital structures such as the globe and orbital nerve. If sufficient removal and reconstruction of the defected area is the goal of the current operation, it is not easy to cover the affected area as much as possible. Our procedure was useful in achieving a sufficient dissection of the fracture site. A learning curve is relatively short.

Our procedure was useful in dissecting the medial wall, and it was also such a safe method as to cause little complications in our clinical series of the patients. Moreover, it leaves nonvisible ancillary subbrow puncture wound. Therefore, it is a recommendable surgical modality.

A complete dissection of the medial orbital wall is always a difficult procedure. However, we assume that our procedure is an efficient approach option to a sufficient dissection of the medial orbital wall through a minimal incision.

\section{NOTES}

\section{Conflict of interest}

No potential conflict of interest relevant to this article was reported.

\section{Ethical approval}

The study was approved by the Institutional Review Board of Konkuk University Hospital (IRB No. KUCH 2017-12-035) and performed in accordance with the principles of the Declaration of Helsinki. Written informed consents were obtained.

\section{Patient consent}

The patients provided written informed consent for the publication and the use of their images.

\section{ORCID}

Seung Min Kim https://orcid.org/0000-0003-2310-2125

Dong In Jo https://orcid.org/0000-0002-3075-4482

Myung Chul Lee https://orcid.org/0000-0002-9721-0092

Soon Heum Kim https://orcid.org/0000-0001-9773-4753

\section{REFERENCES}

1. Chou C, Kuo YR, Chen CC, Lai CS, Lin SD, Huang SH, et al. Medial orbital wall reconstruction with porous polyethylene by using a transconjunctival approach with a caruncular extension. Ann Plast Surg 2017;78(3 Suppl 2):S89-94.

2. Mullins JB, Holds JB, Branham GH, Thomas JR. Complications of the transconjunctival approach: a review of 400 cases. Arch Otolaryngol Head Neck Surg 1997;123:385-8.

3. Kim YH, Park Y, Chung KJ. Considerations for the management of medial orbital wall blowout fracture. Arch Plast Surg 2016;43:229-36.

4. Lee W, Kang DH, Oh SA, Lee SW. Transnasal reduction of blow out fracture with transconjunctival approach. J Korean Cleft Palate Craniofac Assoc 2010;11:1-6.

5. Purnell CA, Vaca EE, Ellis MF. Orbital fracture reconstruction using prebent, anatomic titanium plates: technical tips to avoid complications. J Craniofac Surg 2018;29:e515-7.

6. Chen CT, Huang F, Chen YR. Management of posttraumatic enophthalmos. Chang Gung Med J 2006;29:251-61.

7. Nam SB, Kim KH, Choi SJ, Lee MW, Bae YC. The postoperative results of endoscopic transnasal approach to blowout fractures. J Korean Cleft Palate Craniofac Assoc 2007;8:59-64.

8. Sanno T, Tahara S, Nomura T, Hashikawa K. Endoscopic endonasal reduction for blowout fracture of the medial orbital wall. Plast Reconstr Surg 2003;112:1228-37. 
9. Wang S, Lv J, Xue L, Xi Z, Zheng H, Wang R. Anatomic study and clinical significance of extended endonasal anterior skull base surgery. Neurol India 2014;62:525-31.

10. Choi WK, Kang DH, Oh SA. Anatomical reconstruction of the medial orbital wall fracture. Arch Craniofac Surg 2012;13:2935.

11. Baumann A, Ewers R. Transcaruncular approach for reconstruction of medial orbital wall fracture. Int J Oral Maxillofac Surg 2000;29:264-7.

12. Lim JH, Kim TG, Lee JH, Kim YH. Inlay grafting for the treatment of the posterior comminuted fracture of medial orbital wall. J Korean Cleft Palate Craniofac Assoc 2009;10:55-60.

13. Shi W, Jia R, Li Z, He D, Fan X. Combination of transorbital and endoscopic transnasal approaches to repair orbital medial wall and floor fractures. J Craniofac Surg 2012;23:71-4.

14. Strong EB, Kim KK, Diaz RC. Endoscopic approach to orbital blowout fracture repair. Otolaryngol Head Neck Surg 2004;131: 683-95.

15. Fernandes R, Fattahi T, Steinberg B, Schare H. Endoscopic repair of isolated orbital floor fracture with implant placement. J Oral Maxillofac Surg 2007;65:1449-53.
16. Jeon SY, Kwon JH, Kim JP, Ahn SK, Park JJ, Hur DG, et al. Endoscopic intranasal reduction of the orbit in isolated blowout fractures. Acta Otolaryngol Suppl 2007;127:102-9.

17. Kakibuchi M, Fukazawa K, Fukuda K, Yamada N, Matsuda K, Kawai $\mathrm{K}$, et al. Combination of transconjunctival and endonasal-transantral approach in the repair of blowout fractures involving the orbital floor. Br J Plast Surg 2004;57:37-44.

18. Choi SJ, Oh HC, Nam SB, Kang CU, Bae YC. The reconstruction of the extensive inferior blow-out fracture through endoscopic transnasal and subciliary approaches. J Korean Cleft Palate Craniofac Assoc 2009;10:86-90.

19. Eitzen JP, Elsas FJ. Strabismus following endoscopic intranasal sinus surgery. J Pediatr Ophthalmol Strabismus 1991;28:16870.

20. Hamra ST. Arcus marginalis release and orbital fat preservation in midface rejuvenation. Plast Reconstr Surg 1995;96:35462.

21. Shorr N, Baylis HI, Goldberg RA, Perry JD. Transcaruncular approach to the medial orbit and orbital apex. Ophthalmology 2000;107:1459-63. 